\title{
Higher Serum Asprosin Level is Associated with Urinary Albumin Excretion and Renal Function in Type 2 Diabetes
}

This article was published in the following Dove Press journal: Diabetes, Metabolic Syndrome and Obesity: Targets and Therapy

\section{Xia Deng \\ Li Zhao \\ Chang Guo (D) \\ Ling Yang \\ Dong Wang \\ Yanyan Li \\ Hong Xia \\ Chenxi Wang \\ Zhensheng Cai \\ Lian Li \\ Zhicong Zhao \\ Guoyue Yuan}

Department of Endocrinology, Affiliated Hospital of Jiangsu University, Zhenjiang, jiangsu 2I200I, People's Republic of China

\footnotetext{
Correspondence: Guoyue Yuan; Zhicong Zhao

Department of Endocrinology, Affiliated Hospital of Jiangsu University, 438 Jiefang Road, Zhenjiang, Jiangsu 21200I, People's Republic of China

Tel +86-I3505289352; +86- I87529656I5

Email yuanguoyue@ujs.edu.cn;

2211517025@stmail.ujs.edu.cn
}

\begin{abstract}
Aim: We detected whether serum asprosin levels play a role in the occurrence and development of albuminuria in patients with type 2 diabetes mellitus (T2DM), which has not been previously discussed.

Methods: Based on urinary albumin/creatinine ratio (UACR), 207 T2DM patients were divided into T2DM patients with normoalbuminuria (UACR $<30 \mathrm{mg} / \mathrm{g}$ ), microalbuminuria $(30 \leq \mathrm{UACR}<300 \mathrm{mg} / \mathrm{g}$ ), and macroalbuminuria (UACR $\geq 300 \mathrm{mg} / \mathrm{g}$ ). Serum asprosin levels were determined by enzyme-linked immunosorbent assay.

Results: Comparatively, the serum asprosin levels in T2DM patient groups with macroalbuminuria [2.37 (1.63-3.57)] and microalbuminuria [2.10 (1.60-2.90)] were significantly increased than the normoalbuminuria group [1.59 $(1.18-2.09)](P<0.001)$. Importantly, the serum level of asprosin was positively correlated with UACR $(r=0.304, P<0.001)$, creatinine ( $r=0.157, P=0.024)$, blood urea nitrogen (BUN) $(r=0.244, P<0.001)$, and negatively with glomerular filtration rate (eGFR) $(r=-0.159, P=0.022)$. Furthermore, multiple stepwise regression analyses showed that asprosin was significantly and independently related to UACR, BUN, DBP, and LDL-C $(P<0.05)$. Besides, after adjustment for the confounders, the serum asprosin level was constantly and independently associated with the development of albuminuria in T2DM patients [OR (95\% CI): 2.003 (1.37 2.928), $P<0.001$ ].

Conclusion: Obviously, the serum asprosin level was independently correlated with UACR in T2DM patients, which implies circulating asprosin may play an essential role in the pathogenesis of diabetic nephropathy.
\end{abstract}

Keywords: asprosin, type 2 diabetes mellitus, diabetic nephropathy

\section{Introduction}

Asprosin, a newly discovered protein containing 140 amino acids, was found to be mainly secreted and expressed by white adipose tissue, and then recruited primarily to the liver. ${ }^{1}$ Besides, under hyperlipidemic conditions, $\beta$-cells can also secrete asprosin. $^{2}$ Recent study has pointed out that asprosin plays a crucial role in lipid metabolism and glucose homeostasis. ${ }^{1,3,4}$ Exemplary, several clinical studies reported increased asprosin levels in patients with obesity, type 1 diabetes mellitus (T1DM) and type 2 diabetes mellitus (T2DM). ${ }^{5-7}$ As a novel adipokine, asprosin has demonstrated a positive correlation with adiponectin, and a negative correlation with leptin. ${ }^{8}$ Notably, animal studies suggested that asprosin antibody intervention could significantly enhance glycogen synthesis and improve insulin resistance, thereby reducing blood glucose and insulin levels, and improving glucose 
metabolism. ${ }^{1}$ Also, it has been reported that asprosin antibody reduced not only glucose, but also the weight of obese mice. Further evidence from the mechanistic study demonstrated that asprosin could also cross the bloodbrain barrier and enter the cerebrospinal fluid, and then activate the hypothalamic appetite producing neurons agouti-related protein (AgRP) by regulating the $\mathrm{G}$ protein $\alpha$ subunit (Gas)-Cyclic adenosine monophosphate (cAMP)-dependent protein kinase (PKA) pathway. Meanwhile, in the process of activation, it releases the inhibitory neurotransmitter $\gamma$-aminobutyric acid (GABA), thus, inhibiting the neurons of appetite, proopiomelanocortin (POMC), in order to stimulate appetite and promote the accumulation of weight and obesity. ${ }^{9}$ An in vitro study demonstrated that palmitate enhanced the secretion of asprosin in mouse insulinoma MIN6 cells and human primary islets cells. Moreover, it was documented that recombinant asprosin enhanced the inflammatory response and cell dysfunction in a dose-dependent manner. However, knockdown of asprosin reversed the effect of palmitate, reduced the inflammation of islet cells, and increased glucose-stimulated insulin secretion and cell viability. ${ }^{2}$ In addition, asprosin anticipation could promote the homing of transplanted bone marrow mesenchymal stem cells (MSCs) and significantly increase the left ventricular ejection fraction in the mouse model of myocardial infarction. ${ }^{10}$ The findings mentioned above indicate that asprosin, a metabolic adipokine, can affect glucose and lipid metabolism, energy metabolism and islet cell function. It may participate in the metabolic process through a variety of signaling pathways, and has great potential for application in the diagnosis and treatment of metabolic diseases.

Diabetic Nephropathy (DN) is one of the most important microvascular complications of T2DM and one of the common causes of end-stage renal disease and T2DM death. ${ }^{11}$ Generally, as a metabolic disease, DN occurrence and development are considered to be the result of the combined action of multiple factors, including glucose and lipid metabolism disorders, insulin resistance, multiple cytokine disorders, chronic inflammation, and endothelial dysfunction. $^{12-14}$ The main characteristic feature of DN is impaired glomerular filtration barrier, which is often evaluated by estimated glomerular filtration rate (eGFR). ${ }^{15}$ In the early stage of the DN, clinical symptoms are usually not obvious, showing only glomerular hypertrophy, elevated renal bloodstream, increased glomerular filtration rate, slight morphological change of glomerulus, and the occurrence of microalbuminuria. However, with the progress of the disease, glomerulosclerosis and tubulointerstitial fibrosis develop, and then gradually lead to the deterioration in renal function and macroalbuminuria, and subsequently, developed to end-stage renal disease. ${ }^{12,16}$ Hence, urinary albumin creatinine ratio (UACR) is often used as one of the evaluation indexes of early DN. ${ }^{17,18}$ Due to the lack of early symptoms or signs, some patients have severely renal complications before clinical diagnosis, which seriously threatens the disease control and quality of life of T2DM patients. ${ }^{11}$ Therefore, early prevention and diagnosis of DN is particularly important. ${ }^{19}$

As mentioned above, asprosin is a novel cytokine mainly secreted by the adipose cells. Of note, the expression and secretion of asprosin are affected by the nutritional or metabolic state of the body, and it can regulate the metabolism of glucose and lipid in different degrees. ${ }^{1,3,4}$ Latest report indicated that anti-asprosin can reduce serum glucose, blood urea nitrogen, serum creatinine, and proteinuria, and increase creatinine clearance rate in a dose-dependent manner. More importantly, it can delay the pathophysiological and biochemical progress of kidney in high fat diet/streptozotocin (STZ)-induced type 2 diabetic rat models. ${ }^{20}$ Although accumulated evidence implicated asprosin as an adipokine with powerful metabolic effects, however, little is known about whether circulating asprosin levels are altered in different stages of albuminuria states. Therefore, in this study, we aimed to determine the clinical relevance of asprosin in T2DM patients by measuring its serum concentrations and analyzing for its association with UACR and renal function. Moreover, other clinical indicators of patients with type 2 diabetes were also studied.

\section{Subjects}

In this cross-sectional study, we recruited 264 patients with type 2 diabetes. Patients were diagnosed and treated continuously in the endocrine clinic of Affiliated Hospital of Jiangsu University from August 2016 to August 2019, according to the standards of the American Diabetes Association. ${ }^{21}$ Of note, subjects with the following conditions: 1) other types of diabetes; 2) acute complications of diabetes; 3) chronic virus or bacterial infection; 4) other severe kidney diseases and drug-induced kidney diseases; 5) severe liver diseases; 6) asthma and other autoimmune diseases; 7) tumors; 8) psychosis, etc. were excluded. In addition, in order to reduce the impact of renal failure on asprosin excretion, all T2DM patients 
with estimated glomerular filtration rate (eGFR) less than or equal to $30 \mathrm{~mL} /\left(\min 1.73 \mathrm{~m}^{2}\right)$ were excluded from this research. Finally, 207 patients (mean age $61.6 \pm 10.7$ years) were included in the study. Data on the administration of hypoglycemic drugs, lipid-lowering drugs, and angiotensin-converting enzyme inhibitor (ACEI) and/or angiotensin receptor blockers (ARBs) in the last three months were obtained from the clinical records of each patients. This study was approved by the medical ethics committee of the Affiliated Hospital of Jiangsu University in Zhenjiang, China. All participants had informed consent to the purpose of the study.

Microalbuminuria and Macroalbuminuria were measured by common clinical indicators (UACR). The T2DM patients with an UACR $\leq 30 \mathrm{mg} / \mathrm{g}$ were defined as normoalbuminuria group $(\mathrm{N}=107)$, the T2DM patients with an UACR 30-300 mg/g were defined as microalbuminuria group $(\mathrm{N}=80)$ and then those with an UACR $\geq 300 \mathrm{mg} / \mathrm{g}$ were defined as macroalbuminuria group $(\mathrm{N}=20)$. Renal function was estimated by eGFR using simplified modification of diet in renal disease equation $(\mathrm{sMDRD}), \quad$ as follows: $\quad \mathrm{eGFR}=186.3 \times \quad$ (serum creatinine $)^{-1.154} \times(\text { age })^{-0.203} \times[(0.742) \text { if female }]^{22}$.

\section{Methods}

\section{Clinical and Biochemical Data Collection}

General clinical data of patients, including age, gender, height, weight, blood pressure, history of hypertension, waist circumference (WC), and hip circumference (HC) were collected. WHR was computed as the ratio of WC to $\mathrm{HC}$, and BMI was expressed as weight per square kilometer $(\mathrm{kg} / \mathrm{m} 2)$. After 8 hours of overnight fast, blood samples were extracted from the anterior cubital vein for monitoring various indicators or frozen in a refrigerator at $-80{ }^{\circ} \mathrm{C}$. Next, the Rate method was then used to detect the blood urea nitrogen (BUN), enzymatic method was used to detect the serum creatinine ( $\mathrm{SCr}$ ), UACR was measured by immunoturbidimetry. Glucose oxidase-based assay was used to detect the plasma glucose at each time point of OGTT, and chemiluminescence method was used to measure the insulin and c-peptide level. Glycosylated hemoglobin (HbA1c) was measured by high-performance liquid chromatography (HPLC) (Arkray Inc., Kyoto, Japan). Moreover, the high-density lipoprotein cholesterol (HDLC), low-density lipoprotein cholesterol (LDL-C), total cholesterol (TC), and triglyceride (TG) parameters of blood lipid profile were measured by appropriate enzymatic assays (Beckman Coulter Inc). Serum uric acid was examined by chemiluminescence. Insulin resistance, was estimated by the homeostasis model assessment (HOMA): HOMA-IR $=$ fasting plasma insulin $($ FIns $) \times$ fasting plasma glucose $(\mathrm{FPG}) / 22$. . $^{23}$

\section{Estimation of Serum Asprosin Levels}

The blood samples collected were centrifuged at $1000 \times \mathrm{g}$ at $4{ }^{\circ} \mathrm{C}$ for $20 \mathrm{~min}$ and then each serum sample was separated and labeled. Each serum was kept in an $-80^{\circ} \mathrm{C}$ freezer immediately after collection. Serum asprosin levels were measured by a commercial enzyme-linked immunosorbent assay kit (ELISA) (Jiangsu, Feiya biological technology, Jiangsu, China; catalogue No. MM-1650H1). The sensitivity was $0.1 \mathrm{ng} / \mathrm{mL}$; and the intra- and inter-assay variability was $10 \%$ and $15 \%$, respectively.

\section{Statistical Analysis}

All statistical analyses were performed using SPSS version 20.0. Continuous variables were presented as mean \pm standard deviations $(\bar{x} \pm \mathrm{SD})$ for normally distributed data, and/or medians [inter-quantile range (IQR)] for skew distribution data, and frequency (n) and percentage (\%) for categorical variable data. The data of skewed distribution were statistically analyzed by logarithmic transformation. One-way ANOVA was used to compare the data between groups. Correlation coefficients of the asprosin with the other clinical indices were assessed by Pearson or Spearman correlation analysis in unadjusted model, and were assessed by Partial correlations analysis after adjusted for age, sex, smoking, and drug use. The independent influencing factors of asprosin were determined by multiple stepwise linear regression analysis. Multivariate binary logistic regression model was used to analyze the correlation between asprosin and the development of albuminuria (UACR $\geq 30 \mathrm{mg} / \mathrm{g}$ ).

\section{Results}

\section{Clinical and Biochemical Features in Different Subgroups}

We enrolled 107 (mean age 60.15 \pm 10.56 years, 63 males and 44 females) T2DM with normoalbuminuria, 80 (mean age $62.96 \pm 10.87$ years, 47 males and 33 females) T2DM with microalbuminuria, and 20 (mean age $63.40 \pm 10.51$ years, 10 males and 10 females) T2DM with macroalbuminuria. The prevalence of T2DM complicated with microalbuminuria was $38.6 \%$, and with macroalbuminuria was $9.7 \%$. Detailed 
baseline clinical and biochemical features of all participants are appeared in Table 1. From the result obtained, DM duration, BMI, systolic pressure (SBP), diastolic pressure (DBP), 2-hour postprandial plasma glucose (2hPG), serum uric acid, TG, apoprotein B, percentages of hypertensive and drug use of ACEI or ARB were significantly different among T2DM with normoalbuminuria, microalbuminuria, and macroalbuminuria $(P<0.05)$. Moreover, the T2DM patients with macroalbuminuria and microalbuminuria showed a significant increase UACR levels[655.82 (389.03-1460.65) vs $56.91 \quad(36.75-90.80)$ vs $14.50 \quad(8.45-20.78) \quad \mathrm{mg} / \mathrm{g}$, $P<0.001]$, serum BUN (6.76 (5.03-8.48) vs 5.85 (4.73-7.05) vs $5.33(4.45-6.22) \mathrm{mmol} / \mathrm{L}, P=0.001)$ and creatinine $(79.35$ (61.60-102.13) vs $66.90(54.28-80.08)$ vs 59.90 (49.6069.00) $\mu \mathrm{mol} / \mathrm{L}, P=0.003)$ and a significant decrease in eGFR $(84.36 \pm 34.16$ vs $105.10 \pm 35.80$ vs $116.44 \pm 34.39 \mathrm{~mL} /$ $\left.\left[\min 1.73 \mathrm{~m}^{2}\right], P<0.001\right)$ when compared with those with normoalbuminuria. The ANOVA results of other clinical and

Table I Clinical Characteristics and Asprosin Levels in Diabetic Patients by Categories of Albuminuria

\begin{tabular}{|c|c|c|c|c|}
\hline Characteristics & $\begin{array}{l}\text { T2DM with } \\
\text { Normoalbuminuria }(n=107)\end{array}$ & $\begin{array}{l}\text { T2DM with } \\
\text { Microalbuminuria }(n=80)\end{array}$ & $\begin{array}{l}\text { T2DM with } \\
\text { Macroalbuminuria }(n=20)\end{array}$ & $P$ value \\
\hline Age, y & $60.15 \pm 10.56$ & $62.96 \pm 10.87$ & $63.40 \pm 10.51$ & 0.149 \\
\hline Sex, male (\%) & 63(58.9\%) & $47(58.8 \%)$ & $10(50.0 \%)$ & 0.749 \\
\hline DM duration, mouth & $96.00(12.00-120.00)$ & $120.00(51.00-213.00) *$ & $150.00(21.75,240.00) *$ & 0.006 \\
\hline Smoking, n (\%) & $29(27.1 \%)$ & $22(27.5 \%)$ & $2(10.0 \%)$ & 0.242 \\
\hline BMI & $24.22 \pm 3.45$ & $24.93 \pm 3.43$ & $25.81 \pm 2.84 *$ & 0.025 \\
\hline WHR & $0.92 \pm 0.06$ & $0.94 \pm 0.07$ & $0.95 \pm 0.05$ & 0.065 \\
\hline $\mathrm{SBP}(\mathrm{mmHg})$ & $129.21 \pm 13.33$ & $138.18 \pm 16.36 *$ & $146.35 \pm 17.22 * \dagger$ & $<0.001$ \\
\hline $\mathrm{DBP}(\mathrm{mmHg})$ & $77.88 \pm 7.28$ & $82.21 \pm 9.11 *$ & $82.85 \pm 8.73 *$ & 0.001 \\
\hline Hypertension, n (\%) & $57(53.3 \%)$ & $59(72.5 \%) *$ & $19(95 \%) * \dagger$ & $<0.001$ \\
\hline ACEI or ARB use & $29(27.0 \%)$ & $22(27.5 \%)$ & $10(50.0 \%) * \dagger$ & 0.001 \\
\hline Statins use & $34(31.8 \%)$ & $21(26.3 \%)$ & $5(25.0 \%)$ & 0.654 \\
\hline Only diet and exercise & $6(5.6 \%)$ & $3(3.8 \%)$ & - & 0.557 \\
\hline Insulin & $67(62.6 \%)$ & $57(71.3 \%)$ & $15(75 \%)$ & 0.339 \\
\hline Oral agents & 78 (72.9\%) & $58(72.5 \%)$ & II(55\%) & 0.251 \\
\hline HbAlc (\%) & $8.73 \pm 1.88$ & $9.02 \pm 2.05$ & $8.65 \pm 1.80$ & $0.54 I$ \\
\hline $\mathrm{FPG}(\mathrm{mmol} / \mathrm{L})$ & $9.09 \pm 3.50$ & $10.17 \pm 3.77$ & $9.31 \pm 2.62$ & 0.119 \\
\hline $2 \mathrm{hPG}(\mathrm{mmol} / \mathrm{L})$ & $16.91 \pm 5.34$ & $18.34 \pm 5.95 *$ & $15.18 \pm 4.27 \dagger$ & 0.044 \\
\hline Flns $(\mu \mathrm{lU} / \mathrm{mL})$ & $6.71(4.18-12.26)$ & $9.26(4.55-15.55)$ & $10.14(7.85-11.67)$ & 0.190 \\
\hline FC-P (ng/mL) & $1.60(1.10-2.10)$ & $1.81(1.24-2.37)$ & $1.82(1.23-2.42)$ & 0.111 \\
\hline HOMA-IR & $2.87(1.53-4.55)$ & $3.47(2.07-6.46)$ & $3.82(2.51-5.39)$ & 0.068 \\
\hline Uric acid $(\mu \mathrm{mol} / \mathrm{L})$ & $270.46 \pm 77.16$ & $296.18 \pm 94.38$ & $343.50 \pm 83.58 *$ & 0.002 \\
\hline $\mathrm{TC}(\mathrm{mmol} / \mathrm{L})$ & $4.53 \pm 1.05$ & $4.72 \pm 1.12$ & $4.95 \pm 1.13$ & 0.336 \\
\hline TG (mmol/L) & $1.38(0.98-1.88)$ & $1.59(1.15-2.11)$ & $2.14(1.38-2.84) *+$ & 0.006 \\
\hline HDL-C (mmol/L) & $\mathrm{I} .0 \mathrm{I}(0.83-1.33)$ & $0.99(0.87-1.25)$ & $0.90(0.82-1.04)$ & 0.158 \\
\hline LDL-C (mmol/L) & $2.45 \pm 0.78$ & $2.57 \pm 0.90$ & $2.61 \pm 0.86$ & 0.539 \\
\hline Apoprotein A (g/L) & $1.18 \pm 0.28$ & $1.14 \pm 0.23$ & $1.14 \pm 0.18$ & 0.506 \\
\hline Apoprotein B $(\mathrm{g} / \mathrm{L})$ & $0.92 \pm 0.25$ & $0.96 \pm 0.34$ & $1.11 \pm 0.42 *$ & 0.049 \\
\hline Creatinine $(\mu \mathrm{mol} / \mathrm{L})$ & $59.90(49.60-69.00)$ & $66.90(54.28-80.08)$ & $79.35(61.60-102.13) * \dagger$ & 0.001 \\
\hline BUN (mmol/L) & $5.33(4.45-6.22)$ & $5.85(4.73-7.05) *$ & $6.76(5.03-8.48) * \dagger$ & 0.003 \\
\hline UACR (mg/g) & $14.50(8.45-20.78)$ & $56.91(36.75-90.80) *$ & $655.82(389.03-1460.65) * \dagger$ & $<0.001$ \\
\hline $\begin{array}{l}\text { eGFR }(\mathrm{mL} / \\
[\mathrm{min} 1.73 \mathrm{~m} 2])\end{array}$ & $116.44 \pm 34.39$ & $105.10 \pm 35.80 *$ & $84.36 \pm 34.16 * \dagger$ & $<0.001$ \\
\hline Asprosin $(\mathrm{ng} / \mathrm{mL})$ & $1.59(1.18-2.09)$ & $2.10(1.60-2.90) *$ & $2.37(1.63-3.57) *$ & $<0.001$ \\
\hline
\end{tabular}

Notes: Data are presented as means \pm SD or medians (inter-quantile range (IQR)) for continuous variables and number (percentages) for categorical variables. *Significant $P \leq 0.05$ vs group of T2DM with normoalbuminuria. †Significant $P \leq 0.05$ vs group of T2DM with microalbuminuria.

Abbreviations: DM, diabetes mellitus; SBP, systolic blood pressure; DBP, diastolic blood pressure; BMI, body mass index; WHR, waist-to-hip ratio; FPG, fasting plasma glucose; 2hPG, 2-hour postprandial plasma glucose; Fins, fasting plasma insulin; FC-P, fasting C peptide; HOMA-IR, homeostasis model assessment-insulin resistance index; HbAIc, glycosylated hemoglobin c; TG, triglyceride; TC, total cholesterol; HDL-C, high-density lipoprotein cholesterol; LDL-C, low-density lipoprotein cholesterol; BUN, blood urea nitrogen; UACR, urinary albumin-to-creatinine ratio; eGFR, estimated glomerular filtration rate; ACEl, angiotensin-converting enzyme inhibitors; ARB, angiotensin receptor blocker. 
biochemical indices were not statistically significant, as shown in Table 1.

\section{Serum Asprosin Levels in Different Subgroups}

Upon the assessments of serum asprosin levels in the three groups of T2DM patients, the serum asprosin level was 1.59 (1.18-2.09)ng/mL for normoalbuminuria, 2.10 (1.60-2.90) $\mathrm{ng} / \mathrm{mL}$ for microalbuminuria, and 2.37 (1.63-3.57)ng/mL for macroalbuminuria with statistically significant $(P<0.05)$ (Table 1) difference among the groups.

\section{Clinical and Biochemical Features of the Participants According to the Tertiles of Asprosin}

From the results obtained, we observed that subjects in the upper serum asprosin tertile had longer duration, larger proportion of $\mathrm{UACR} \geq 30 \mathrm{mg} / \mathrm{g}$, and higher UACR, BUN, BMI, DBP, FIns, fasting C-peptide (FC-P), serum uric acid, TG, HOMA-IR compared with subjects in the middle or lower serum asprosin tertile $(P<0.05$ or $P<0.01)$ (Table 2).

\section{Correlations Between Serum Asprosin and Other Clinical Variables}

Serum asprosin concentrations positively correlated with UACR, creatinine, BUN, BMI, SBP, DBP, FC-P, serum uric acid, TC, TG, and LDL-C, and negatively correlated with eGFR. After adjustment for age, sex, smoking, and drug use, the UACR, creatinine, BUN, eGFR, DBP, FC-P, uric acid, and LDL-C remained significantly correlated with serum asprosin levels (Table 3). Furthermore, multiple stepwise regression analysis showed the asprosin was significantly and positively independent on UACR, BUN, DBP, and LDL-C $(P<0.05)$ (Table 4$)$.

\section{Logistic Regression Analyses for Microalbuminuria in T2DM Patients}

Binary logistic regression analyses (Table 5) showed the association of asprosin with microalbuminuria (UACR $\geq 30 \mathrm{mg} / \mathrm{g})$ in T2DM patients $(P<0.001$ in every model). Serum asprosin level was significantly correlated with the development of microalbuminuria [OR (95\% CI):1.954 (1.357 2.812), $P<0.001]$ without any adjustment. As illustrated in the model 1, after adjustment for age and sex (Table 5, model 1), the OR for asprosin [OR (95\% CI):
1.953 (1.357 2.812), $P<0.001]$ were significantly related to the development of microalbuminuria. Upon additional adjustments for smoking and drug use (Table 5, model 2), the OR clearly increased to [OR (95\% CI): 2.019 (1.382 2.952), $P<0.001]$. Nevertheless, adjustment for HOMA-IR and BMI did not greatly weaken the association of asprosin with microalbuminuria (Table 5, model 3). The OR for asprosin in model 3 was [OR (95\% CI): 1.931 (1.341 2.781), $P<0.001]$. Moreover, by further adjustment for age, sex, smoking, drug use, BMI, and HOMA-IR (Table 5, model 4), we observed that the OR for DM duration [OR (95\% CI): 1.007 (1.003 1.011), $P=0.001]$ and SBP[OR (95\% CI): 1.055 (1.029 1.082), $P<0.001]$ were significantly related to the development of microalbuminuria, while asprosin had the higher OR and significantly correlated with the development of microalbuminuria [OR (95\% CI): 2.003 (1.37 2.928), $P<0.001]$.

\section{ROC Curve for Predict Microalbuminuria}

Table 6 shows the ROC curve to predict microalbuminuria (UACR $\geq 30 \mathrm{mg} / \mathrm{g}$ ) with selected parameters. Value of AUC (95\% Confidence intervals) were 0.695 (0.623 0.767) for asprosin, $0.640(0.565 \sim 0.716)$ for $\mathrm{DM}$ duration, 0.686 (0.614 0.758) for SBP, $0.614(0.537 \sim 0.691)$ for TG, 0.528 (0.449 0.608) for HAB1c and $0.548(0.469 \sim 0.627)$ for TC, respectively. When 1.93 of asprosin was set as a cut off point for presence of microalbuminuria, the early DN diagnosis revealed a sensitivity of $63.6 \%$ and specificity of $71.7 \%$.

\section{Discussion}

Microalbuminuria can be defined as an early clinical indicator of DN and a predictor of cardiovascular mortality and morbidity. ${ }^{17,24}$ In this study, we investigated the correlation between asprosin, a newly adipokine that regulates metabolic processes, and albuminuria in T2DM patients. We found that serum asprosin level elevated in pace with UACR in T2DM patients, which has never been reported before. Importantly, after adjustment for the confounders, asprosin levels were found to be independently related to albuminuria in T2DM patients. Thus, asprosin may play an important role in the pathogenesis of diabetic nephropathy.

In the present study, serum asprosin levels significantly elevated in T2DM patients with macroalbuminuria and microalbuminuria group as compared with normoalbuminuria group. Notably, the ascended serum asprosin level was associated with elevated serum creatinine and descended eGFR levels in T2DM patients. In addition, the 
Table 2 Clinical and Biochemical Characteristics of the Study Subjects According to the Tertiles of Asprosin

\begin{tabular}{|c|c|c|c|c|}
\hline Characteristics & Lower Tertile $(n=69)$ & Middle Tertile $(n=69)$ & Upper Tertile $(n=69)$ & $P$ value \\
\hline Age, y & $62.99 \pm 10.94$ & $59.83 \pm 10.95$ & $61.84 \pm 10.19$ & 0.216 \\
\hline Sex, male (\%) & $42(60.9 \%)$ & $34(49.3 \%)$ & $44(63.8 \%)$ & 0.189 \\
\hline DM duration, mouth & $96.00(12.00-168.00)$ & $96.00(14.50-168.00)$ & $120.00(54.00,186.00) * \dagger$ & 0.006 \\
\hline Smoking, n (\%) & |4(20.3\%) & $15(21.7 \%)$ & $24(34.8 \%)$ & 0.099 \\
\hline BMI & $24.09 \pm 3.47$ & $24.42 \pm 3.22$ & $25.43 \pm 3.45 *$ & 0.008 \\
\hline WHR & $0.93 \pm 0.07$ & $0.93 \pm 0.05$ & $0.93 \pm 0.06$ & 0.682 \\
\hline $\mathrm{SBP}(\mathrm{mmHg})$ & $132.86 \pm 15.27$ & $133.07 \pm 16.33$ & $137.07 \pm 16.14$ & 0.218 \\
\hline $\mathrm{DBP}(\mathrm{mmHg})$ & $78.13 \pm 7.76$ & $79.35 \pm 7.86$ & $82.62 \pm 9.08 * \dagger$ & 0.005 \\
\hline Hypertension, n (\%) & $37(53.6 \%)$ & $44(63.8 \%)$ & $53(76.8 \%) *$ & 0.017 \\
\hline ACEI or ARB use & $19(27.5 \%)$ & $16(23.2 \%)$ & $26(37.7 \%)$ & 0.159 \\
\hline Statins use & $24(34.8 \%)$ & $17(24.6 \%)$ & $19(27.5 \%)$ & 0.400 \\
\hline Only diet and exercise & $4(5.8 \%)$ & $2(2.9 \%)$ & $3(4.3 \%)$ & 0.706 \\
\hline Insulin & $47(68.1 \%)$ & $4 I(59.4 \%)$ & $5 \mathrm{I}(73.9 \%)$ & 0.189 \\
\hline Oral agents & $44(63.8 \%)$ & $55(79.7 \%)$ & $48(69.6 \%)$ & 0.113 \\
\hline $\mathrm{HbAlc}(\%)$ & $8.92 \pm 2.06$ & $8.81 \pm 1.88$ & $8.78 \pm 1.88$ & 0.541 \\
\hline FPG (mmol/L) & $9.34 \pm 3.76$ & $9.70 \pm 3.36$ & $9.56 \pm 3.58$ & 0.634 \\
\hline $2 \mathrm{hPG}(\mathrm{mmol} / \mathrm{L})$ & $17.57 \pm 5.55$ & $|7.2| \pm 5.6 \mid$ & $17.11 \pm 5.60$ & 0.776 \\
\hline Flns $(\mu \mathrm{IU} / \mathrm{mL})$ & $6.56(4.24-12.51)$ & $6.71(4.04-11.52)$ & $10.14(5.6-18.70) * \dagger$ & 0.008 \\
\hline FC-P (ng/mL) & $1.58(1.05-1.97)$ & $1.80(1.23-2.29) *$ & $1.90(1.19-2.38) *$ & 0.027 \\
\hline HOMA-IR & $2.95(1.42-4.50)$ & $2.70(1.58-4.40)$ & $3.78(2.39-6.77) * \dagger$ & 0.006 \\
\hline Uric acid $(\mu \mathrm{mol} / \mathrm{L})$ & $272.15 \pm 89.53$ & $278.96 \pm 86.45$ & $311.25 \pm 81.69 *$ & 0.005 \\
\hline $\mathrm{TC}(\mathrm{mmol} / \mathrm{L})$ & $4.53 \pm 1.06$ & $4.55 \pm 1.09$ & $4.84 \pm 1.11$ & 0.176 \\
\hline TG $(\mathrm{mmol} / \mathrm{L})$ & $\mid .31(0.94-\mid .8 I)$ & $1.52(1.20-2.03)$ & $1.69(1.17-2.45) *$ & 0.016 \\
\hline HDL-C (mmol/L) & $1.00(0.85-1.27)$ & $1.00(0.83-1.27)$ & $0.99(0.84-1.17)$ & 0.165 \\
\hline LDL-C (mmol/L) & $2.37 \pm 0.76$ & $2.53 \pm 0.87$ & $2.64 \pm 0.86$ & 0.792 \\
\hline Apoprotein A (g/L) & $1.18 \pm 0.27$ & $1.16 \pm 0.24$ & $1.13 \pm 0.23$ & 0.450 \\
\hline Apoprotein B $(\mathrm{g} / \mathrm{L})$ & $0.95 \pm 0.29$ & $0.92 \pm 0.28$ & $1.00 \pm 0.35$ & 0.258 \\
\hline Creatinine $(\mu \mathrm{mol} / \mathrm{L})$ & $60.90(50.15-74.45)$ & $61.70(51.00-77.95)$ & $67.20(54.35-82.30)$ & 0.150 \\
\hline BUN (mmol/L) & $5.42(4.45-6.69)$ & $5.28(4.65-6.59)$ & $6.07(4.84-7.24)$ & 0.113 \\
\hline UACR (mg/g) & $21.33(12.17-35.62)$ & $21.7 I(|2.3|-53.89)$ & $50.78(26.25-117.26) * \dagger$ & $<0.001$ \\
\hline UACR $\geq 30 \mathrm{mg} / \mathrm{g}, \mathrm{n}$ (\%) & $21(30.43 \%)$ & $30(43.48 \%)$ & $49(71.01 \%) * \dagger$ & $<0.001$ \\
\hline eGFR(mL/[min $1.73 \mathrm{~m} 2])$ & $115.06 \pm 36.15$ & $106.12 \pm 35.86$ & $105.70 \pm 35.95$ & 0.228 \\
\hline
\end{tabular}

Notes: *Significant $P \leq 0.05$ vs group of lower tertile. ${ }^{\dagger}$ Significant $P \leq 0.05$ vs group of middle tertile.

serum asprosin concentrations were positively correlated with UACR, creatinine, BUN, and negatively correlated with eGFR even after adjustment for age, sex, smoking, and drug use. Importantly, from the result obtained, no matter how confounding factors were controlled, asprosin in T2DM patients was independently associated with the development of albuminuria. Indeed, the current findings corroborate with a recent study performed by Zhang et $\mathrm{al}^{25}$ in which serum asprosin was found to be elevated in patients with T2DM combined with microalbuminuria. In that study, only 105 subjects were recruited, including: 1) normal glucose tolerance (NGT, $\mathrm{n}=30$ ); 2) type 2 diabetes (T2DM, n = 42); 3) T2DM with diabetic kidney disease (DKD, $\mathrm{n}=33$ ), however, they did not include the patients with macroalbuminuria. Therefore, it is only concluded that asprosin was related to early $\mathrm{DN}$, and the changes of circulating asprosin levels in different albuminuria stages cannot be determined. Nevertheless, the results obtained herein has filled in this gap. In addition, our results further revealed that asprosin showed better diagnostic value in ROC curve used to predict microalbuminuria in T2DM subjects. Notwithstanding, in an animal study conducted by El Kattawy et al $^{20}$ type 2 diabetic rats administered with anti-asprosin antibody significantly reduced the serum urea, serum creatinine and proteinuria compared to control rats. In addition, renal pathology results showed that after anti-asprosin antibody was administered, chronic inflammatory cells around the glomeruli and tubules were slightly aggregated, and the degree of kidney disease was significantly improved. Cumulatively, these results reveal 
Table 3 The Correlations Between Asprosin and Clinical Indices in T2DM Patients

\begin{tabular}{|c|c|c|c|c|}
\hline \multirow[t]{2}{*}{ Characteristics } & \multicolumn{2}{|c|}{ Unadjusted } & \multicolumn{2}{|c|}{$\begin{array}{l}\text { Adjusted for Age, Sex, } \\
\text { Smoking, and Drug Use }\end{array}$} \\
\hline & $r$ & $P$ & $r$ & $P$ \\
\hline Age & 0.022 & 0.755 & - & - \\
\hline Sex & 0.000 & 0.997 & - & - \\
\hline DM duration & 0.120 & 0.084 & 0.154 & 0.093 \\
\hline BMI & 0.191 & 0.006 & 0.096 & 0.180 \\
\hline WHR & 0.055 & 0.434 & 0.056 & 0.543 \\
\hline SBP & 0.139 & 0.045 & 0.116 & 0.105 \\
\hline DBP & 0.223 & 0.001 & 0.253 & $<0.001$ \\
\hline HbAlc & 0.010 & 0.883 & 0.019 & 0.793 \\
\hline FPG & 0.033 & $0.64 I$ & 0.034 & 0.632 \\
\hline $2 h P G$ & -0.031 & 0.662 & 0.003 & 0.970 \\
\hline Flns & 0.134 & 0.054 & 0.060 & 0.401 \\
\hline FC-P & 0.180 & 0.009 & 0.150 & 0.035 \\
\hline HOMA-IR & 0.133 & 0.056 & 0.075 & 0.292 \\
\hline Uric acid & 0.209 & 0.003 & 0.147 & 0.039 \\
\hline TC & 0.146 & 0.036 & 0.118 & 0.098 \\
\hline TG & 0.192 & 0.006 & 0.118 & 0.098 \\
\hline HDL-C & -0.038 & 0.584 & -0.069 & 0.336 \\
\hline LDL-C & 0.186 & 0.007 & 0.142 & 0.046 \\
\hline Apoprotein A & -0.080 & 0.253 & -0.086 & 0.350 \\
\hline Apoprotein B & 0.021 & 0.768 & 0.030 & 0.679 \\
\hline Creatinine & 0.157 & 0.024 & 0.153 & 0.032 \\
\hline BUN & 0.244 & $<0.001$ & 0.151 & 0.034 \\
\hline UACR & 0.304 & $<0.001$ & 0.278 & $<0.001$ \\
\hline eGFR & -0.159 & 0.022 & -0.151 & 0.034 \\
\hline
\end{tabular}

that asprosin may be involved in the occurrence and development of diabetic nephropathy. However, the mechanisms underlying increased asprosin levels in T2DM and DN remain elusive.

It is well established that dyslipidemia may promote the development of DN. ${ }^{26,27}$ Mechanistic study showed that the accumulation of lipids could lead to advanced glycation end products (AGEs) and then increased inflammatory cytokines in the kidneys, resulting in endothelial dysfunction of T2DM, glomerulosclerosis and tubular interstitial damage. ${ }^{28-30}$ Moreover, excessively high blood lipid levels will lead to increased blood viscosity, reducing blood flow in the kidneys, causing renal ischemia, hypoxia, damage to glomerular capillaries and increased permeability, which can further lead to the leakage of albumin and thereby aggravates the occurrence of DN. ${ }^{31}$ Additionally, clinical studies have shown that microalbuminuria was closely related to TG and LDL$\mathrm{C}^{32}$ whereas the early DN was mainly elevated by TG and LDL-C. In fact, as DN progresses, the level of TG and LDL-C obviously increases with increased TC, and a decreased HDL-C. ${ }^{33}$ Likewise, our results showed that the levels of TC, TG and LDL-C in the microalbuminuria group and the macroalbuminuria group were higher, and the HDL-C level was lower than those in the normoalbuminuria group, although the levels of TG, LDL-C, HDL-C among the three groups were not statistically significant. Previous study purported asprosin as a metabolic regulator that plays an important role in lipid metabolism. ${ }^{3}$ In the current study, we observed that BMI, serum TG, and LDL$\mathrm{C}$ level increased along with asprosin in diabetic patients. Serum asprosin concentrations were positively correlated with BMI, TG, TC, and LDL-C, and LDL-C was an independent risk factor influencing serum asprosin levels. In agreement with our findings, other studies have also revealed that asprosin levels were positively correlated with LDL and TG, and negatively correlated with HDL$\mathrm{C}$ in obesity and diabetes subjects. ${ }^{6,7}$ Therefore, dyslipidemia may be involved in the role of asprosin in diabetic nephropathy.

Recent studies have shown that insulin resistance plays an important role in the development of DN. ${ }^{14,34}$ These studies suggested that insulin may contribute to the occurrence and development of DN through mechanisms such as causing hyperinsulinemia, inducing oxidative stress,

Table 4 Stepwise Multiple Linear Regression Analysis with Asprosin as the Dependent Variable

\begin{tabular}{|l|l|l|l|l|l|}
\hline Independent Variable & Regression Coefficient (SE) & Standardized Coefficients Beta & $\boldsymbol{t}$ & $\boldsymbol{P}$ & $\mathbf{9 5 \%} \mathbf{C l}$ \\
\hline (Constant) & $1.570(0.776)$ & - & -2.022 & 0.045 & $-3.101-(-0.039)$ \\
UACR & $0.001(0.000)$ & 0.189 & 2.834 & 0.005 & $0.000-0.001$ \\
BUN & $0.114(0.037)$ & 0.206 & 3.093 & 0.002 & $0.041-0.186$ \\
DBP & $0.027(0.009)$ & 0.195 & 3.044 & 0.003 & $0.010-0.045$ \\
LDL-C & $0.279(0.094)$ & 0.191 & 2.974 & 0.003 & $0.094-0.464$ \\
\hline
\end{tabular}

Notes: The following independent variables were considered for the model: age, sex, smoking, DM duration, BMI, WHR, HbAIc, FPG, 2hPG, Flns, FC-P, TC, TG, LDL-C, HDL-C, HOMA-IR, BUN, Creatinine, eGFR, and drug use. Only the variables that had a $P<0.05$ were considered in the final fitted model.

Abbreviation: $\mathrm{Cl}$, confidence interval. 
Table 5 OR $(95 \% \mathrm{Cl})$ by Binary Logistic Regression Models for Albuminuria with UACR as Dependent Variable

\begin{tabular}{|c|c|c|c|c|c|}
\hline \multirow[t]{3}{*}{ Models } & \multirow[t]{3}{*}{ Covariates } & \multicolumn{4}{|l|}{$\operatorname{UACR}(n=207)$} \\
\hline & & \multicolumn{2}{|c|}{ Normoalbuminuria $(n=107)$} & \multicolumn{2}{|c|}{ Albuminuria $(n=100)$} \\
\hline & & OR (95\% Cl) & $P$ & OR $(95 \% \mathrm{Cl})$ & $P$ \\
\hline \multirow[t]{6}{*}{ Unadjusted } & Asprosin & I & - & $\mathrm{I} .954(\mathrm{I} .357 \sim 2.8 \mathrm{I} 2)$ & $<0.001$ \\
\hline & DM duration & I & - & $\mathrm{I} .006(\mathrm{I} .002 \sim 1.0 \mathrm{I})$ & 0.001 \\
\hline & SBP & I & - & $\mathrm{I} .05 \mathrm{I}(\mathrm{I} .027 \sim 1.076)$ & $<0.001$ \\
\hline & HABIC & 1 & - & I.074(0.908 1.272) & 0.404 \\
\hline & TG & I & - & I.I42(0.899 I.452) & 0.277 \\
\hline & $\mathrm{TC}$ & 1 & - & $0.979(0.706 \sim 1.357)$ & 0.898 \\
\hline \multirow[t]{6}{*}{ Model I } & Asprosin & 1 & - & $1.953(\mathrm{I} .357 \sim 2.8 \mathrm{I} 2)$ & $<0.001$ \\
\hline & DM duration & I & - & $\mathrm{I} .006(\mathrm{I} .002 \sim 1.0 \mathrm{I})$ & 0.002 \\
\hline & SBP & 1 & - & $1.05(1.025 \sim 1.076)$ & $<0.00$ I \\
\hline & HABIC & I & - & $1.079(0.91 \sim 1.279)$ & 0.381 \\
\hline & TG & I & - & $1.15 \mathrm{I}(0.90 \mathrm{I} \sim \mathrm{I} .47)$ & 0.259 \\
\hline & TC & 1 & - & $0.988(0.707 \sim 1.38)$ & 0.943 \\
\hline \multirow[t]{6}{*}{ Model 2} & Asprosin & I & - & $2.019(1.382 \sim 2.952)$ & $<0.001$ \\
\hline & DM duration & I & - & $1.007(1.003 \sim 1.01)$ & 0.001 \\
\hline & SBP & 1 & - & $1.056(1.03 \sim 1.082)$ & $<0.001$ \\
\hline & $\mathrm{HABIC}$ & I & - & I.059(0.884 1.269) & 0.533 \\
\hline & TG & I & - & $1.229(0.955 \sim 1.58 \mathrm{I})$ & 0.109 \\
\hline & TC & I & - & $0.903(0.644 \sim 1.267)$ & 0.556 \\
\hline \multirow[t]{6}{*}{ Model 3} & Asprosin & I & - & $\mathrm{I} .93 \mathrm{I}(\mathrm{I} .34 \mathrm{I} 2.78 \mathrm{I})$ & $<0.001$ \\
\hline & DM duration & I & - & $1.006(1.003 \sim 1.01)$ & 0.001 \\
\hline & SBP & I & - & $1.05(1.026 \sim 1.075)$ & $<0.00$ I \\
\hline & HABIC & I & - & $1.093(0.918 \sim 1.303)$ & 0.317 \\
\hline & TG & I & - & $1.096(0.86 \sim 1.397)$ & 0.460 \\
\hline & $\mathrm{TC}$ & I & - & $0.968(0.69 \sim 1.358)$ & 0.853 \\
\hline \multirow[t]{6}{*}{ Model 4} & Asprosin & I & - & $2.003(1.37 \sim 2.928)$ & $<0.001$ \\
\hline & DM duration & 1 & - & $\mathrm{I} .007(\mathrm{I} .003 \sim 1.0 \mathrm{II})$ & 0.001 \\
\hline & SBP & 1 & - & I.055(I.029 1.082) & $<0.001$ \\
\hline & HABIC & I & - & I.074(0.892 1.294) & 0.450 \\
\hline & TG & I & - & I.I77(0.904 I.53) & 0.226 \\
\hline & $\mathrm{TC}$ & I & - & $0.905(0.635 \sim 1.291)$ & 0.582 \\
\hline
\end{tabular}

Notes: Model I adjusted for age and sex, Model 2 adjusted for smoking and drug use, Model 3 adjusted for BMI and HOMA-IR, Model 4 adjusted for age, sex, smoking, drug use, BMI, and HOMA-IR. $P<0.05$ was considered to be statistically significant.

and activating inflammatory factors. In the current study, subjects in the upper asprosin tertile had higher FIns, FC$\mathrm{P}$, and HOMA-IR compared with subjects in the middle or lower serum asprosin tertile. This seems to be consistent with previous clinical studies. According to a study conducted by Wang et al, ${ }^{35}$ circulating asprosin level was positively correlated with HOMA-IR, and negatively correlated with acute insulin response (AIR), HOMA- $\beta$, and area under the curve of insulin secretion (AUC) in T2DM patients. Groener et $\mathrm{al}^{5}$ performed hyperinsulinemichypoglycemic clamp and intravenous glucose tolerance test in T1DM patients. Their experimental results showed that patients with elevated plasma asprosin level during initial hypoglycemia or hypoglycemia showed higher overall insulin sensitivity, and plasma asprosin level was positively correlated with serum insulin concentration and basic insulin dose to fat free mass. Furthermore, in patients with polycystic ovary syndrome, Alan et $\mathrm{al}^{36}$ found a positive correlation between asprosin level and insulin resistance. Interestingly, an in vivo and vitro study demonstrated the effect of aspirin on insulin resistance. In that study, asprosin reduced insulin-stimulated phosphorylation of insulin receptor substrate 1 and protein kinase B (Akt), as well as glucose uptake in skeletal muscle $\mathrm{C} 2 \mathrm{C} 12$ cell, 
Table 6 Receiver Operating Characteristic Curves for the Ability of Asprosin to Discriminate the Presence of Albuminuria in Patients with Type 2 Diabetes

\begin{tabular}{|l|l|l|l|}
\hline Variables & AUC & $\mathbf{9 5 \%} \mathbf{C l}$ & $\boldsymbol{P}$ \\
\hline Asprosin & 0.695 & $0.623 \sim 0.767$ & $<0.00 \mathrm{I}$ \\
DM duration & 0.640 & $0.565 \sim 0.716$ & $0.00 \mathrm{I}$ \\
SBP & 0.686 & $0.614 \sim 0.758$ & $<0.00 \mathrm{I}$ \\
HABIC & 0.528 & $0.449 \sim 0.608$ & $0.48 \mathrm{I}$ \\
TG & 0.614 & $0.537 \sim 0.69 \mathrm{I}$ & 0.005 \\
TC & 0.548 & $0.469 \sim 0.627$ & 0.238 \\
\hline
\end{tabular}

Abbreviation: AUC, area under the curve.

and subsequently aggravated the deterioration of insulin sensitivity. Further animal experiments showed that asprosin intervention resulted in impaired glucose tolerance and insulin resistance. In addition, knockdown of asprosin reduced the impaired glucose tolerance and then insulin resistance induced by high-fat diet. Furthermore, mechanistic study found that asprosin caused Protein kinase $\mathrm{C} \delta$ (PKC 8 )/Sarco-ER Ca2+-ATPase(SERCA)2 mediated endoplasmic reticulum (ER) stress and inflammation, which led to the impairment of insulin signal in skeletal muscle cells. ${ }^{37}$ Another in vitro study also confirmed that asprosin could upregulated Toll-like receptor (TLR) 4/ cJun-N-terminal-kinase (JNK) signaling pathway, leading to $\beta$-cell inflammation, cell dysfunction, and decreased insulin secretion. However, asprosin knockout reversed the effect of palmitate, reduced the inflammatory response of islet cells, and increased glucose-induced insulin secretion and cell viability. ${ }^{2}$ Thence, there are reasons to believe that asprosin may participate in diabetic nephropathy through insulin resistance.

Consequently, the present study did not find any correlation between serum asprosin and plasma glucose level, which is inconsistence with the results of the previous studies. ${ }^{3,7,25}$ However, the cause of these discrepancies is still unclear, which may be attributed partly to the different stages of diabetes and then the type of drugs used. This study has the following limitations. Firstly, UACR was used as a surrogate variable for diabetic nephropathy, but it was not the gold standard. Although we excluded patients with other possible acute or chronic kidney disease based on clinical and biochemical examination results, ideally, the diagnosis of diabetic nephropathy should be confirmed by renal biopsy. Secondly, the present study was a cross-sectional study, so we could not determine the exact causal relationship between the elevated serum asprosin level and albuminuria in T2DM patients. Finally, we excluded patients with eGFR less than or equal to $30 \mathrm{~mL} /\left(\min 1.73 \mathrm{~m}^{2}\right)$ to minimize the effect of renal failure on asprosin. Therefore, despite the above limitations, we can still conclude that asprosin is significantly positively associated with UACR in T2DM patients.

\section{Conclusion}

In conclusion, our study has pinpointed out that serum asprosin levels were significantly increased in T2DM patients with regard to the different stages of albuminuria, especially in macroalbuminuric patients. Subjects in the upper serum asprosin tertile had higher UACR as well as higher proportions of UACR $\geq 30 \mathrm{mg} / \mathrm{g}$, BUN, FIns, FC-P, and TG, HOMAIR compared with subjects in the middle or lower serum asprosin tertile. Asprosin positively correlated with UACR, creatinine, BUN, BMI, SBP, DBP, FC-P, TC, TG, and LDL$\mathrm{C}$, whereas it was inversely correlated with eGFR. Moreover, after adjustment for the confounders, the level of asprosin was constantly independent on the development of albuminuria in T2DM patients. Combined with previous studies, we speculated that the asprosin may participate in the process of diabetic nephropathy by affecting dyslipidemia and insulin resistance. Further research is needed to clarify the potential pathophysiological role of asprosin in diabetic nephropathy.

\section{Compliance with Ethical Standards}

The study was approved by the Biomedical Research Ethics Committee of Affiliated Hospital of Jiangsu University, Zhenjiang, China, and performed in accordance with the Declaration of Helsinki. All participants had informed consent to the purpose of the study.

\section{Funding}

This work was supported by the National Natural Science Foundation of China (81870548 and 81570721), the Social Development Project of Jiangsu Province (BE2018692), the Natural Science Foundation of Jiangsu Province, China (BK20191222), the High Caliber Medical Personnel Foundation of Jiangsu Province (LGY2016053), the Six Talent Peaks Project in Jiangsu Province (2015-WSN -006), and the Fifth "169 project" Scientific Research Project of Zhenjiang City, Jiangsu Province. The Scientific Research Projects of Jiangsu Health and Family Planning Commission (Y2018109).

\section{Disclosure}

The authors declared no potential competing interests with respect to the research, authorship, and/or publication of this article. 


\section{References}

1. Romere C, Duerrschmid C, Bournat J, et al. Asprosin, a fasting-induced glucogenic protein hormone. Cell. 2016;165 (3):566-579. doi:10.1016/j.cell.2016.02.063

2. Lee T, Yun S, Jeong JH, Jung TW. Asprosin impairs insulin secretion in response to glucose and viability through TLR4/JNK-mediated inflammation. Mol Cell Endocrinol. 2019;486:96-104. doi:10.1016/ j.mce.2019.03.001

3. Li X, Liao M, Shen R, et al. Plasma asprosin levels are associated with glucose metabolism, lipid, and sex hormone profiles in females with metabolic-related diseases. Mediators Inflamm. 2018;2018:7375294. doi:10.1155/2018/7375294

4. Yuan M, Li W, Zhu Y, Yu B, Wu J. Asprosin: a novel player in metabolic diseases. Front Endocrinol (Lausanne). 2020;11:64. doi:10.3389/fendo.2020.00064

5. Groener JB, Valkanou A, Kender Z, et al. Asprosin response in hypoglycemia is not related to hypoglycemia unawareness but rather to insulin resistance in type 1 diabetes. PLoS One. 2019;14(9): e0222771. doi:10.1371/journal.pone.0222771

6. Ugur K, Aydin S. Saliva and blood asprosin hormone concentration associated with obesity. Int $J$ Endocrinol. 2019;2019:2521096. doi: $10.1155 / 2019 / 2521096$

7. Zhang L, Chen C, Zhou N, Fu Y, Cheng X. Circulating asprosin concentrations are increased in type 2 diabetes mellitus and independently associated with fasting glucose and triglyceride. Clin Chim Acta. 2019;489:183-188. doi:10.1016/j.cca.2017.10.034

8. Wiecek M, Szymura J, Maciejczyk M, Kantorowicz M, Szygula Z. Acute anaerobic exercise affects the secretion of asprosin, irisin, and other cytokines - a comparison between sexes. Front Physiol. 2018;9:1782. doi:10.3389/fphys.2018.01782

9. Duerrschmid C, He Y, Wang C, et al. Asprosin is a centrally acting orexigenic hormone. Nat Med. 2017;23(12):1444-1453. doi:10.1038/ nm. 4432

10. Zhang Z, Tan Y, Zhu L, et al. Asprosin improves the survival of mesenchymal stromal cells in myocardial infarction by inhibiting apoptosis via the activated ERK1/2-SOD2 pathway. Life Sci. 2019;231:116554. doi:10.1016/j.1fs.2019.116554

11. Tuttle KR, Bakris GL, Bilous RW, et al. Diabetic kidney disease: a report from an ADA consensus conference. Diabetes Care. 2014;37 (10):2864-2883. doi:10.2337/dc14-1296

12. Reidy K, Kang HM, Hostetter T, Susztak K. Molecular mechanisms of diabetic kidney disease. J Clin Invest. 2014;124(6):2333-2340. doi:10.1172/JCI72271

13. Aghadavod E, Khodadadi S, Baradaran A, Nasri P, Bahmani M, Rafieian-Kopaei M. Role of oxidative stress and inflammatory factors in diabetic kidney disease. Iran J Kidney Dis. 2016;10(6):337-343.

14. Gnudi L, Coward RJM, Long DA. Diabetic nephropathy: perspective on novel molecular mechanisms. Trends Endocrinol Metab. 2016;27 (11):820-830. doi:10.1016/j.tem.2016.07.002

15. Doshi SM, Friedman AN. Diagnosis and management of type 2 diabetic kidney disease. Clin J Am Soc Nephrol. 2017;12 (8):1366-1373. doi:10.2215/CJN.11111016

16. Alicic RZ, Rooney MT, Tuttle KR. Diabetic kidney disease: challenges, progress, and possibilities. Clin J Am Soc Nephrol. 2017;12 (12):2032-2045. doi:10.2215/cjn.11491116

17. Stehouwer CD, Gall MA, Twisk JW, Knudsen E, Emeis JJ, Parving HH. Increased urinary albumin excretion, endothelial dysfunction, and chronic low-grade inflammation in type 2 diabetes: progressive, interrelated, and independently associated with risk of death. Diabetes. 2002;51(4):1157-1165. doi:10.2337/diabetes.51.4.1157

18. Ninomiya T, Perkovic V, de Galan BE, et al. Albuminuria and kidney function independently predict cardiovascular and renal outcomes in diabetes. J Am Soc Nephrol. 2009;20(8):1813-1821. doi:10.1681/ ASN.2008121270
19. Afkarian M, Zelnick LR, Hall YN, et al. Clinical manifestations of kidney disease among US adults with diabetes, 1988-2014. JAMA. 2016;316(6):602-610. doi:10.1001/jama.2016.10924

20. El Kattawy HA, Ashour WM. Anti-asprosin: a potential protective role against the progression of diabetic nephropathy in type 2 diabetic rats. Am J Biomed Sci. 2019;11(3).

21. American Diabetes A. (2) classification and diagnosis of diabetes. Diabetes Care. 2015;38(Suppl):S8-S16. doi:10.2337/dc15-S005

22. Ma YC, Zuo L, Chen JH, et al. Modified glomerular filtration rate estimating equation for Chinese patients with chronic kidney disease. J Am Soc Nephrol. 2006;17(10):2937-2944. doi:10.1681/ASN.2006 040368

23. Antuna-Puente B, Disse E, Rabasa-Lhoret R, Laville M, Capeau J, Bastard JP. How can we measure insulin sensitivity/resistance? Diabetes Metab. 2011;37(3):179-188. doi:10.1016/j.diabet.2011. 01.002

24. Sarafidis PA, Bakris GL. Microalbuminuria and chronic kidney disease as risk factors for cardiovascular disease. Nephrol Dial Transplant. 2006;21(9):2366-2374. doi:10.1093/ndt/gfl309

25. Zhang $\mathrm{H}, \mathrm{Hu} \mathrm{W}$, Zhang G. Circulating asprosin levels are increased in patients with type 2 diabetes and associated with early-stage diabetic kidney disease. Int Urol Nephrol. 2020;52(8):1517-1522. doi:10.1007/s11255-020-02509-8

26. Toyama T, Shimizu M, Furuichi K, Kaneko S, Wada T. Treatment and impact of dyslipidemia in diabetic nephropathy. Clin Exp Nephrol. 2014;18(2):201-205. doi:10.1007/s10157-013-0898-1

27. Bonacina F, Baragetti A, Catapano AL, Norata GD. The interconnection between immuno-metabolism, diabetes, and CKD. Curr Diab Rep. 2019;19(5):21. doi:10.1007/s11892-019-1143-4

28. Zhang Y, Ma KL, Liu J, et al. Inflammatory stress exacerbates lipid accumulation and podocyte injuries in diabetic nephropathy. Acta Diabetol. 2015;52(6):1045-1056. doi:10.1007/s00592-015-0753-9

29. Lieben L. Diabetic nephropathy: lipid toxicity drives renal disease. Nat Rev Nephrol. 2017;13(4):194. doi:10.1038/nrneph.2017.22

30. Yuan Y, Sun H, Sun Z. Advanced glycation end products (AGEs) increase renal lipid accumulation: a pathogenic factor of diabetic nephropathy (DN). Lipids Health Dis. 2017;16(1):126. doi:10.1186/ s12944-017-0522-6

31. Ng KF, Aung HH, Rutledge JC. Role of triglyceride-rich lipoproteins in renal injury. Contrib Nephrol. 2011;170:165-171. doi:10.1159/ 000325654

32. Islam N, Kazmi F, Chusney GD, Mattock MB, Zaini A, Pickup JC. Ethnic differences in correlates of microalbuminuria in NIDDM. The role of the acute-phase response. Diabetes Care. 1998;21 (3):385-388. doi:10.2337/diacare.21.3.385

33. Hirano T. Abnormal lipoprotein metabolism in diabetic nephropathy. Clin Exp Nephrol. 2014;18(2):206-209. doi:10.1007/s10157-0130880-y

34. Wei W, An XR, Jin SJ, Li XX, Xu M. Inhibition of insulin resistance by PGE1 via autophagy-dependent FGF21 pathway in diabetic nephropathy. Sci Rep. 2018;8(1):9. doi:10.1038/s41598-017-18427-2

35. Wang Y, Qu H, Xiong X, et al. Plasma asprosin concentrations are increased in individuals with glucose dysregulation and correlated with insulin resistance and first-phase insulin secretion. Mediators Inflamm. 2018;2018:9471583. doi:10.1155/2018/9471583

36. Alan M, Gurlek B, Yilmaz A, et al. Asprosin: a novel peptide hormone related to insulin resistance in women with polycystic ovary syndrome. Gynecol Endocrinol. 2019;35(3):220-223. doi:10.1080/09513590.2018.1512967

37. Jung TW, Kim HC, Kim HU, et al. Asprosin attenuates insulin signaling pathway through PKCdelta-activated ER stress and inflammation in skeletal muscle. $J$ Cell Physiol. 2019;234 (11):20888-20899. doi:10.1002/jcp.28694 


\section{Publish your work in this journal}

Diabetes, Metabolic Syndrome and Obesity: Targets and Therapy is an international, peer-reviewed open-access journal committed to the rapid publication of the latest laboratory and clinical findings in the fields of diabetes, metabolic syndrome and obesity research. Original research, review, case reports, hypothesis formation, expert opinion and commentaries are all considered for publication. The manuscript management system is completely online and includes a very quick and fair peer-review system, which is all easy to use. Visit http://www.dovepress.com/testimonials.php to read real quotes from published authors. 